\title{
Continuing medical education in Radiology in South Africa
}

In her 2009 presidential address, RSNA President Theresa McLoud ${ }^{1}$ focused on trends in Radiological Training: National and International Implications. She said: 'We need to embrace the change that globalization is bringing and actively join in the discussion as we seek ways to adapt ourselves and our training programs to it.'

The editorial in the June 2010 issue of this journal focused positively on the state of academic radiology in South Africa and also drew attention to the continuing medical education (CME) programme of the Radiological Society of South Africa (RSSA).

The RSSA is a professional society whose purpose is to improve the health of patients through maximising the value of radiology and radiologists by:

- advancing the science of radiology

- improving radiological service to patients

- studying the socio-economic aspects of the practice of radiology

- providing and supporting continuing education for radiologists and related professional fields.

The RSSA also supports its members as they adapt to a rapidly changing healthcare environment. The challenges for the RSSA are complex because the needs of patients are diverse. To best represent and fulfill the CME requirements, the RSSA Congress Portfolio must relate to many different organisations and entities.

The RSSA had for decades before the introduction by the HPCSA in 2007 of the obligatory continuing professional development (CPD) programme, organised national and international congresses and courses. I pay tribute to Dr Paul Schneider, Peter Reichman, Professor Brian Cremin, Professor Jan Lotz, Dr Jan Labuschagne and others who contributed to the congress programme over many years. I vividly recall lectures by internationally renowned radiologists such as Drs Margulis, Levine, Laufer, Husband, Modick, Maffee, Resnick, Osborn, Bradey, Harnsberger, Heiken, Kucharczyk and many other great names.

Despite such events, there are most likely very few private radiologists who have not regularly travelled overseas to attend congresses and courses at great expense. Against the background of optimism regarding the state of academic radiology in South Africa, most teaching departments are sub-optimally staffed, and no single department can boast a full complement of sub/super-specialists or fellowship-trained faculty staff. Few full-time staff or registrars can afford to regularly travel abroad to attend congresses and courses, unless they participate academically, which is not always possible. However, I believe that if all resources are pooled and effectively managed, and the wide spectrum of local expertise accessed, the RSSA can significantly contribute to the training and CME needs of the profession.

Against this background, I accepted the nomination of RSSA Congress Chair. The vision was to establish an active, integrated, affordable and sustainable state-of-the-art CME programme that will address and complement the academic needs (shortfall) of registrars in training, as well as the ongoing CME requirements of academic and private radiologists.
The programme was launched with the full support of the RSSA Exco and many other role players. From February 2008 to August 2010, there have been 10 courses including 2 short courses on Current Ethical Issues in Radiology. In addition, there were separate workshops by SAMSIG, the RSSA-affiliated Musculo-Skeletal Sub-group, the Paediatric Imaging Society (PISA) and a RSSA-endorsed PACS course. More than 40 international speakers have participated so far. All this was achieved by forming partnerships and securing RSSA participation in the satellite and outreach educational programmes of international societies such as the International Cancer Imaging Society (ICIS), International Society for Magnetic Resonance and Medicine (ISMRM) Global Outreach Program, Radiological Society of North America (RSNA), Visiting International Professors Program(VIP), the Society of Gastro-Intestinal Radiology (SGR), International Education Conference (IEC), the University of Leuven and leading international radiologists.

On an international intersociety level, RSSA members have free corresponding membership of the ECR, and registrars and junior radiologists qualify for participation in the European School of Radiology visiting fellowship programmes.The International Society of Radiology (ISR) allows free access by RSSA members, academic institutions and registrars to an online virtual journal project, Gorad, which gives online access to 17 selected major journals for free. Course topics are chosen and integrated to cover the major organ system and imaging modalities over a 4-year period. Ideally, all the academic departments in the country should have a similar integrated modular curriculum, and the CME congress/course programme should be an integral part of the curriculum. It is anticipated that registrars should be exposed to the leading teachers in radiology during their training and that practicing radiologists would have completed the 'Essentials for board certification review. All courses are available on CD for participants. Every effort is made to promote faculty participation and chair opportunities for South African radiologists, and to create the opportunity to participate and gain experience at an international level. Great care is taken to keep the courses affordable and registration fees market-related, compared with similar courses overseas. Registrars and full-time academic staff are cross-subsidised by private radiologists. It is, however, important to establish a culture of investing in oneself among young colleagues.

The CME programme can only be sustained over time if it runs at a profit. The financial support of the major vendors and pharmaceutical companies is indispensible for the programme's success, and is highly appreciated. Long-term partnerships with international radiological societies and commercial vendors must be continuously fostered and maintained. New and innovative approaches to industry and corporate sponsorships and education grants will have to be formulated and agreed upon by all stakeholders, to pool the limited and available financial resources for the benefit of all. It is important to take cognisance of the 'winds of change' about international rules and trends in corporate

continued on page 78 